\title{
Absorption enhancement in graphene with an efficient resonator
}

\author{
Xiao, Binggang; Gu, Mingyue; Qin, Kang; Xiao, Sanshui
}

Published in:

Optical and Quantum Electronics

Link to article, DOI:

$10.1007 / \mathrm{s} 11082-017-1013-8$

Publication date:

2017

Document Version

Peer reviewed version

Link back to DTU Orbit

Citation (APA):

Xiao, B., Gu, M., Qin, K., \& Xiao, S. (2017). Absorption enhancement in graphene with an efficient resonator. Optical and Quantum Electronics, 49(5), [177 ]. https://doi.org/10.1007/s11082-017-1013-8

\section{General rights}

Copyright and moral rights for the publications made accessible in the public portal are retained by the authors and/or other copyright owners and it is a condition of accessing publications that users recognise and abide by the legal requirements associated with these rights.

- Users may download and print one copy of any publication from the public portal for the purpose of private study or research.

- You may not further distribute the material or use it for any profit-making activity or commercial gain

- You may freely distribute the URL identifying the publication in the public portal

If you believe that this document breaches copyright please contact us providing details, and we will remove access to the work immediately and investigate your claim. 


\title{
Absorption Enhancement in Graphene with an Efficient Resonator
}

\author{
Binggang Xiao, Mingyue Gu, Kang Qin, Sanshui Xiao
}

\begin{abstract}
Graphene can be utilized in designing tunable terahertz (THz) devices due to its tunability of sheet conductivity, suffering however with weak light-graphene interactions. In this paper, an absorption enhancement in graphene using a Fabry-Perot resonator is presented, and its performance has been numerically investigated using finite element method (FEM). The Fabry-Perot resonator consists of a continuous layer of graphene film sandwiched between the polymethyl methacrylate (PMMA) and silicon layers on an Au substrate which is covered by periodic gold ribbons. Numerical results show that the absorption performance is significantly enhanced by use of the Fabry-Perot resonator and a narrow band perfect absorption is achieved in THz regime. The influence of structural parameters on the absorption performance is further analyzed, and the absorption peak frequency can be flexibly controlled by adjusting the chemical potential of graphene which could be conveniently achieved by applying a bias voltage. The proposed structure here has a promising potential for developing advanced $\mathrm{THz}$ optics-electronics devices.
\end{abstract}

Index Terms - Absorber, Graphene, $\mathrm{THz}$

\section{INTRODUCTION}

Graphene as a two-dimensional material has been a hot topic in recent years, since a team led by Novoselov and Geim successfully separated graphene from graphite in an efficient way in 2004 [1]. Graphene is a single layer of carbon atoms arranged in plane with a honey comb lattice. Due to its unique electric and optical properties, such as high carrier mobility,

This work was supported by 2016 Zhejiang Provincial Natural Science Foundation under Grant No.LY16F010010, in part by 2015 Zhejiang Province Public Welfare of International Cooperation Project under Grant No. 2015 C34006.

Binggang Xiao, Mingyue Gu, Kang Qin are with the College of Information Engineering, China Ji Liang University, hangzhou 310018, China (e-mail: bgxiao@cjlu.edu.cn,984804997@qq.com,492883770@qq.com.)

Sanshui Xiao is with the Department of Photonic Engineering, Technical University of Demark, DTU Osterds Plads, Copenhagen, DK 2800 (e-mail: saxi@ fotonik.dtu.dk). zero-gap bandstructure and tunability of conductivity by applying bias voltage or chemical doping, graphene has become a very promising material [2-4] for the development of modulators and ultrafast lasers. In a wide range of frequencies, the absorption of monolayer graphene amounts to $\pi \alpha \approx 2.3 \%$, with $\alpha=1 / 137$ being the fine structure constant $[5,6]$. The weak light-matter interaction leads to $97.7 \%$ of light unaffected by the presence of the graphene layer. Consequently, the efficiency of graphene-based optoelectronic devices is strongly limited by the weak light-graphene interactions. In order to improve its absorption efficiency, one has combined graphene into optical cavities [7], dielectric gratings [8], and photonic crystals $[9,10]$ where electromagnetic wave can be efficiently trapped and absorbed. Recently, there are many work using different shapes of graphene to enhance the absorption of electromagnetic wave, such as graphene nanodisks [11], graphene open micro-ring arrays [12] or graphene micro-ribbon [13] and periodically elliptical nanodisk graphene structure [14].

In this paper, we investigate a simply and efficient perfect absorber by use of Fabry-Perot resonator structure in the $\mathrm{THz}$ regime. By changing the geometric parameters of the structure and adjusting the chemical potential of graphene, we can achieve the perfect absorption.

\section{CAlculation METHOD}

The graphene was numerically modeled by a thin layer (with thickness $\Delta=1 \mathrm{~nm}$ ) with the relative permittivity of $\varepsilon_{\mathrm{GR}}$ $=1+i \sigma_{\mathrm{g}} / \omega \Delta \varepsilon_{0}$, where $\sigma_{\mathrm{g}}$ is the conductivity of graphene sheet derived using the well-known Kubo formula [15]. The conductivity of the graphene is described with interband and intraband contributions as follows:

$\sigma_{g}=\sigma_{\text {int } r a}\left(\omega, \mu_{c}, \Gamma, \mathrm{T}\right)+\sigma_{\text {int } e r}\left(\omega, \mu_{c}, \Gamma, \mathrm{T}\right)$

$\sigma_{\text {inter }} \approx \frac{-i e^{2}}{4 \pi \hbar} \ln \left[\frac{2\left|\mu_{c}\right|-(\omega-i 2 \Gamma) \hbar}{2\left|\mu_{c}\right|+(\omega-i 2 \Gamma) \hbar}\right]$ 


$$
\sigma_{\text {intra }}=\frac{-i e^{2} K_{B} T}{\pi \hbar^{2}(\omega-i 2 \Gamma)}\left[\frac{\mu_{c}}{K_{B} T}+2 \ln \left(e^{-\mu_{c} / K_{B} T}+1\right)\right]
$$

where $e, \hbar$ and $K_{B}$ are electron charge, reduced Plank's and Boltzmann's constant, respectively. $\omega$ is the angular frequency, $\mu_{\mathrm{c}}$ is chemical potential, $T$ is the temperature and fixed to $300 \mathrm{~K}$, and $\Gamma$ is the scattering rate, which is related to relaxation time $\tau$ $(\tau=1 / 2 \Gamma)$.

For $\mathrm{THz}$ frequencies, where the photon energy $\hbar \omega \leq E_{F}, E_{F}$ $\geqslant \mathrm{k}_{\mathrm{B}} \mathrm{T}$, the interband part is negligible comparing to the intraband part. Therefore, in the $\mathrm{THz}$ regime, graphene is well described by the Drude-like conductivity with Eq. (3). In addition, the frequency dependent permittivity of graphene depending on the some selected values of the chemical potential are shown in Fig. 1(a) and 1(b).
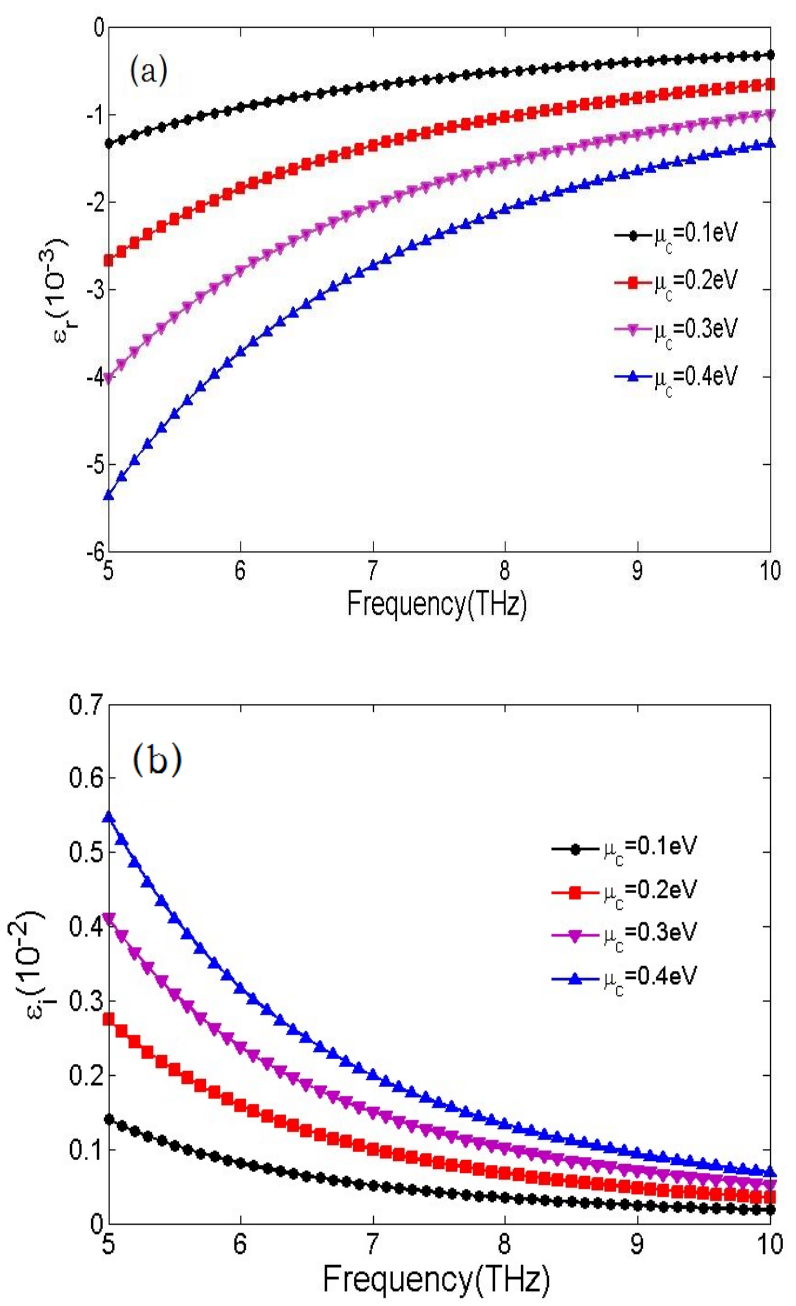

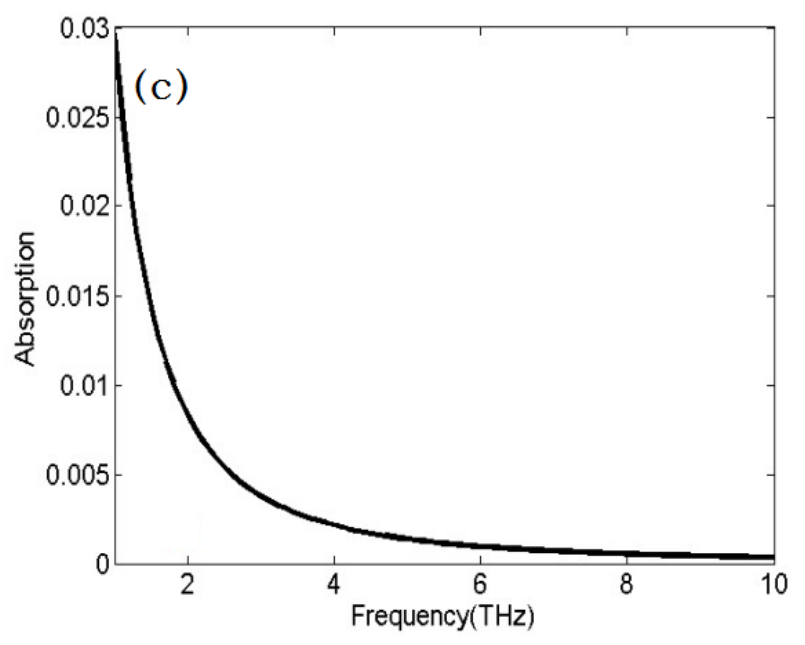

Fig. 1. Real (a) and imaginary (b) parts of the relative permittivity of a $1 \mathrm{~nm}$-thickness graphene sheet as function of frequency as well as chemical potential. (c) The absorption efficiency of monolayer graphene in terahertz ranges.

Fig. 1(a) clearly shows that the real part of graphene relative permittivity is negative in the $\mathrm{THz}$ range, it means that the graphene sheet have the property of metal. When the chemical potential increases from $0.1 \mathrm{eV}$ to $0.4 \mathrm{eV}$, the amplitudes of real part and imaginary part of relative permittivity become larger (the absolute value of the real part becomes larger) as shown in Fig. 1(a) and 1(b), so the chemical potential has a significant effect on graphene. Although graphene owns the property of metal in terahertz regime, the absorbance is still very low as shown in Fig. 1(c). We can see that with the frequency increases, the absorption efficiency decreases obviously in the $\mathrm{THz}$ range, it's due to the graphene in high frequency has the smaller dielectric loss. Compared with noble metal, graphene has many excellent properties. For example, graphene suffers lower loss than noble metal because of its smaller imaginary part of relative permittivity. And, even if the geometrical structure is fixed, we can adjust the absorption frequency and amplitude by static voltage. In conclusion, graphene becomes a potential candidate to take the place of noble metal in the $\mathrm{THz}$ regime.

\section{SIMULATION RESULTS AND DISCUSSION}

In this paper, the proposed structure was shown in the Fig. 2(a), the top layer is the periodic Au strips. In order to understand the influence of geometrical parameters on the absorption effect, the width $\mathrm{w}$ of top layer Au strip of proposed structure is discussed, ranging from $5.5 \mu \mathrm{m}$ to $7 \mu \mathrm{m}$. And the thickness of middle layer PMMA is changing from $300 \mathrm{~nm}$ to $700 \mathrm{~nm}$. It's worth noting that the thickness of Au is bigger than skin depth in the $\mathrm{THz}$ regime. So the reflection is the only factor limiting the absorption efficiency. In the discussion, we analyze the one parameter under the circumstance of fixing another parameter in order to choose optimal value according to simulation. In the paper, relative permittivity of PMMA and $\mathrm{Si}$ 
is 2.3 and 11.6 respectively, the conductivity of $\mathrm{Au}$ is $4.7 \times 10^{\wedge} 7$ $\mathrm{S} / \mathrm{m}$, which is perfectly reflecting in the $\mathrm{THz}$ frequency region. In our calculation, the numerical simulations are conducted using finite element technique in Comsol MultiPhysics. FEM accompanied with periodic boundary condition has been employed to calculate the absorption spectrum of the structure. The total absorption can be calculated by: $A=1-T-R$ ( $T$ and $R$ is transmission and reflection coefficient respectively). The incident plane wave is considered to be normal to the proposed structure along the $\mathrm{z}$ direction. The absorption enhancement of graphene in the proposed structure can be explained through analyzing electric and magnetic field distributions, as shows in Figs. 3(a)-3(d). At the absorption peak of $6.78 \mathrm{THz},|\mathrm{E}|$ is confined in the middle layer PMMA between graphene and metal gold strips as shown in Fig. 3(a). And $|\mathrm{H}|$ is between gold ribbons and gold substrate as shown in Fig. 3(b). In contrast, at $6.02 \mathrm{THz}$, as shown in Fig. 3(c) and 3(d), the filed is very low. Our structure can be seen as a Fabry-Perot resonator, which is similar to metal-insulator-metal (MIM) structure [16].

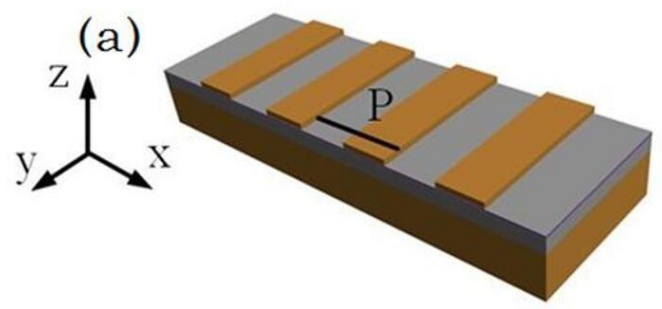

(b)

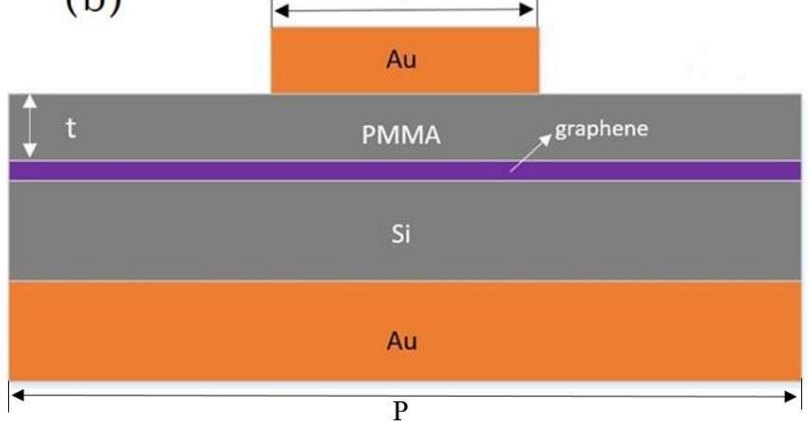

Fig. 2. (a) 3D schematic of the proposed structure and (b) 2D schematic of the proposed structure, with Au $0.5 \mu \mathrm{m}$ thick, Si $3 \mu \mathrm{m}$ thick and periodic $\mathrm{P}=25 \mu \mathrm{m}$.


Fig. 3. Field distributions for the TM polarized, where $\mathrm{w}=7 \mu \mathrm{m}, \mathrm{t}=0.5 \mu \mathrm{m}$.

Electric field (a) and magnetic field (b) at $\mathrm{f}=6.78 \mathrm{THz}$.

Electric field (c) and magnetic field (d) at $\mathrm{f}=6.02 \mathrm{THz}$.

Different from [13], our structure is relatively easy to be fabricated. Because of our structure doesn't need to tailor the graphene strip. Besides, compared to metal-based absorber [13, 18], graphene-based absorber is easily tunable. Fig. 4 shows the absorption performance of the proposed structure at different geometric parameters. Firstly, the width of metal Au strip is discussed when the other geometric parameters is fixed. Fig. 4(a) shows that the absorption spectra of the TM-polarized incident wave with four different widths $w$ of the metal stripes ranging from $5.5 \mu \mathrm{m}$ to $7 \mu \mathrm{m}$. Here we set the period of the structure as $25 \mu \mathrm{m}$, and thickness of the PMMA is $500 \mathrm{~nm}$. The solid lines represent the absorption spectrum with bottom metallic substrate, and the dashed lines for the case without bottom metallic substrate. From Fig. 4(a) we can see that the absorption efficiency is very low without the gold substrate, the absorption efficiency is just ranging from $30 \%$ to $38 \%$. When having the gold substrate, absorption efficiency is enhanced significantly. This is due to the fact that the incident electromagnetic wave is reflected by metallic substrate, so the electromagnetic wave pass through the graphene film with multiple times, thus enhancing the absorption in graphene. As $\mathrm{w}$ increases from $5.5 \mu \mathrm{m}$ to $7.0 \mu \mathrm{m}$, the absorption spectra of graphene are red-shifted. Here the edges of the Au strips act as sources of surface plasmon-polaritons, which are induced by the underlying graphene sheet, and constructive interference results in the resonance dependence on the Au width [19]. That is to say, absorption frequencies of graphene metamaterial can be decided by the width of Au strip. So we can easily tune the absorption peak by changing the width of Au strip. 

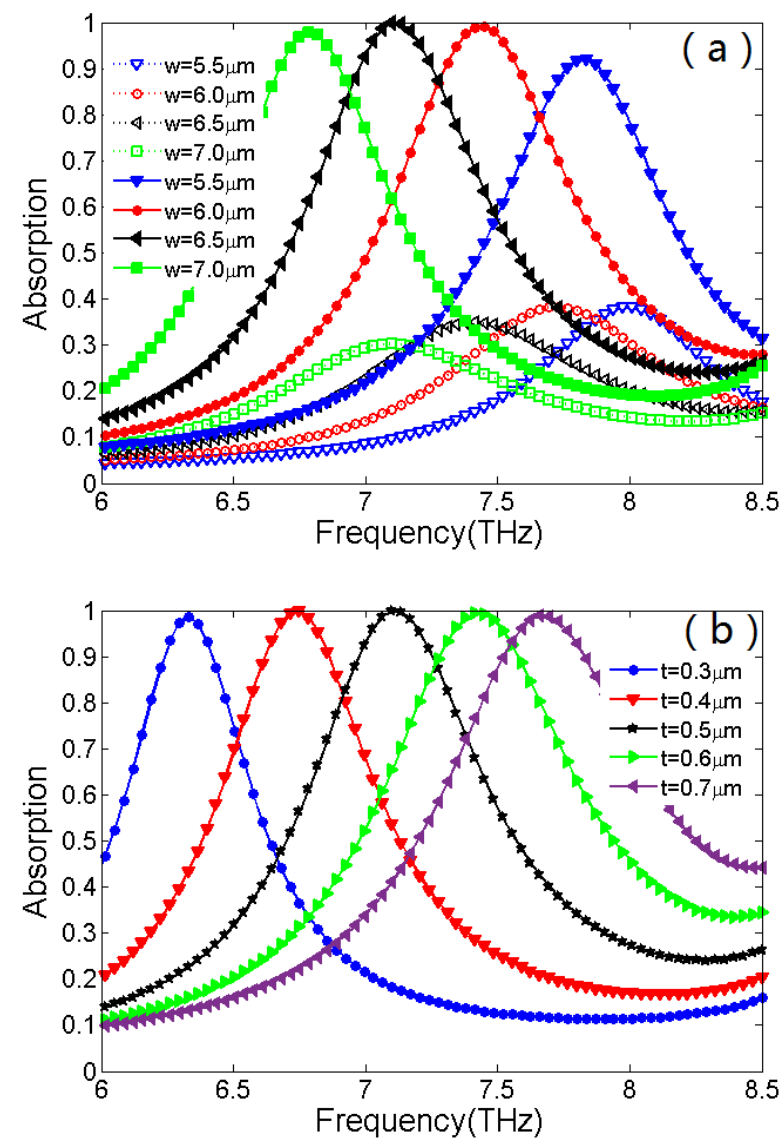

Fig. 4. Absorption spectra where $\mathrm{p}=25 \mu \mathrm{m}, \mu_{\mathrm{c}}=0.1 \mathrm{eV}$. (a) for different $\mathrm{w}$ values with (the solid lines) and without (the dashed lines) the bottom metallic substrate where $\mathrm{t}=500 \mathrm{~nm}$. (b) for different $\mathrm{t}$ values, where $\mathrm{w}=6.5 \mu \mathrm{m}$.

Lastly, we discuss the dependence of absorption spectra on working frequency at different thickness of PMMA. Similar to MIM structure in the optical regime, the absorption spectra of the structure is sensitive to thickness of PMMA. As shown in the Fig. 4(b), as the thickness of PMMA increases from 300nm to $700 \mathrm{~nm}$, the absorption spectrum shows obvious resonances peaks, and the peaks shift from $6.35 \mathrm{THz}$ to $7.70 \mathrm{THz}$ due to the increase of the resonant gap. And the absorption peak is above $90 \%$, the possible reason is that coupling between graphene and metal Au become strong. Near field coupling existing between the graphene and Au strip lead to the energy confined in the middle layer PMMA.

Besides the geometric parameter can affect the absorption peak, we can also tune the chemical potential to change the performance of the proposed structure. As we know, the chemical potential has a great impact on the conductivity of graphene, which can be controlled by electrostatic and chemical doping [20, 21]. As shown in the Fig. 5, absorption spectrum has been calculated at different chemical potential (or Fermi level). The frequency of the absorption resonance experiences the blue shift with the increase of chemical potential. It should be mentioned that almost perfect absorption can be achieved in the tuning range. Therefore, by applying a gate voltage on the graphene layer, the chemical potential and thus the conductivity of graphene can be controlled on purpose.

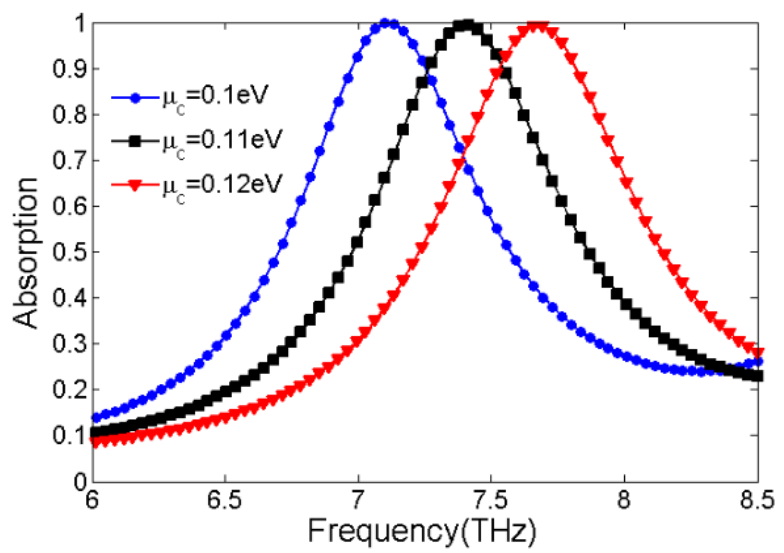

Fig. 5. Absorption spectra at different chemical potential $\mu_{\mathrm{c}}$, where we set $\mathrm{P}=25 \mu \mathrm{m}, \mathrm{w}=6.5 \mu \mathrm{m}, \mathrm{t}=0.5 \mu \mathrm{m}$

\section{CONCLUSION}

We have proposed a perfect absorber with tunable resonance frequency by employing a Fabry-Perot structure. The absorption performance is numerically investigated by the finite element method, and the absorption efficiency can be improved from $40 \%$ to above $90 \%$ by the present of the resonator. Simulation results show that the absorption peak frequency can be adjusted in the terahertz frequency by tuning the bias voltage on the graphene layer. The influence of geometrical parameters on the absorption is also analyzed systemically. The research achievements reveal that our work has provided an efficient method to manipulate the $\mathrm{THz}$ wave which could be used in many $\mathrm{THz}$ devices such as modulators, sensors, and detectors, etc.

\section{REFERENCES}

[1] Geim, A. K., Novoselov, K.S.: The rise of graphene. Nature Mater. 6 (3), 183-91 (2007).

[2] Sun, Z., Martinez, A., Wang, F.: Optical modulators with 2D layered materials. Nat. Photon. 10, 227-238 (2016).

[3] Xiao, S., Zhu, X., Li, B-H., Mortensen, N. A.: Graphene-plasmon polaritons: From fundamental properties to potential applications, Front. Phys. 11, 117801 (2016).

[4] Ding, Y., Zhu, X., Xiao, S., Hu, H., Frandsen, L. H., Mortensen, N. A., Yvind, K.: Effective electro-optical modulation with high extinction ratio by a graphene-silicon microring resonator. Nano Lett. 15, 4393-4400 (2015).

[5] Nair, R. R., Blake, P., Grigorenko, A. N., Novoselov, K. S., Booth, T. J., Stauber, T., Peres, N. M. R., Geim, A. K.: Fine structure constant defines visual transparency of graphene. Science, 320(5881):1308-1308 (2008).

[6] Stauber T., Peres, N. M. R., Geim, A. K.: The optical conductivity of graphene in the visible region of the spectrum. Phys. Rev. B, 78, 085432 (2008). 
[7] Ferreira, A., Peres, N. M. R., Ribeiro, R. M. Stauber, T.: Efficient graphene-based photodetector with two cavities. Phys. Rev. B, 85, 115438 (2012)

[8] Zhan, T. R., Zhao, F. Y., Hu, X. H., Liu, X. H., Zi, J.: Band structure of plasmons and optical absorption enhancement in graphene on subwavelength dielectric gratings at infrared frequencies. Phys. Rev. B 86, 165416 (2012).

[9] Piper, J. R., Fan, S. H.:. Total Absorption in a Graphene Monolayer in the Optical Regime by Critical Coupling with a Photonic Crystal Guided Resonance. ACS Photonics, 1(4) 347-353 (2014).

[10] Liu, J. T., Liu, N. H., Li, J. Li, X. J., Huang, J-H: Enhanced absorption of graphene with one-dimensional photonic crystal. Appl. Phys. Lett., 101, 052104 (2012).

[11] Thongrattanasiri, S., Koppens F. H. L., Garcia de Abajo, F. J.: Complete optical absorption in periodically patterned graphene. Phys. Rev. Lett., 108, 047401 (2012).

[12] Gao, R., Xu, Z., Ding, C. Wu, L., Yao, J.: Graphene metamaterial for multiband and broadband terahertz absorber. Opt. Commun., 356, 400-404 (2015).

[13] Alaee, R., Farhat, M., Rockstuhl, C., Lederer, F.: A perfect absorber made of a graphene micro-ribbon metamaterial. Optics Express, 20(27), 28017-24 (2012).

[14] Yao, G., Ling, F, Yue, J., Luo, C.. Ji, J, Yao, J.: Dual-band tunable perfect metamaterial absorber in the THz range. Optics Express, 24(2), 1518-1527 (2016).

[15] Hanson, G. W.: Dyadic Green's Functions and Guided Surface Waves on Graphene. Appl. Phys. Lett.,103, 064302 (2008).

[16] Nielsen, M.G., Gramotnev, D.K., Pors, A., Albrektsen, O., Bozhevolnyi, S.I.: Continuous layer gap plasmon resonators. Optics Express, 19(20), 19310-19322 (2011).

[17] Landy, N. I., Bingham, C. M., Tyler, T., Jokerst, N., Smith, D. R., Padilla, W. J.: Design, theory, and measurement of a polarization-insensitive absorber for terahertz imaging. Phys. Rev. B, 79, 125104 (2009).

[18] Ma, Y., Che.n Q., Grant, J. Saha, S. C., Khalid, A., Cumming, D. R. S.: A terahertz polarization insensitive dual band metamaterial absorber. Opt. Lett., 36(6), 945-947 (2011).

[19] Bludov, Y. V., Ferreira, A., Peres, N. M. R., Vasilevskiy, M. I. : A primer on surface plasmon-polaritons in graphene. International Journal of Modern Physics B, 27(10), 1341001 (2013).

[20] Fang, Z., Wang, Y., Schlather, A. E., Liu, Z., Ajayan, P. M., Garcia de Abajo, F. J., Nordlander, P., Zhu, X., Halas, N. J.: Active tunable absorption enhancement with graphene nanodisk arrays. Nano Lett., 14(1), 299-304 (2014).

[21] Fang, Z., Thongrattanasiri, S., Schlather, A., Liu, Z., Ma, L., Wang, Y., Ayayan, P. M., Nordlander, P., Halas, N. J., Garcia de Abajo, F. J.: Gated tunability and hybridization of localized plasmons in nanostructured graphene. ACS Nano, 7(3), 2388-2395 (2013). 